\title{
Stroke and Major Bleeding when Switching from Warfarin to Apixaban in Patients with Advanced Chronic Kidney Disease and Prevalent Atrial Fibrillation
}

Katherine Garlo ( $\sim$ katherine.garlo@gmail.com )

Brigham and Women's Hospital

Thomas Mavrakanas

McGill University

Wei Wang

Harvard University

Elisabeth Burdick

Brigham and Women's Hospital

David Charytan

New York University Langone Medical Center

\section{Research Article}

Keywords: Chronic kidney disease, atrial fibrillation, anticoagulation, stroke, major bleeding, risk, benefit

Posted Date: January 19th, 2021

DOI: https://doi.org/10.21203/rs.3.rs-144190/v1

License: (c) (i) This work is licensed under a Creative Commons Attribution 4.0 International License.

Read Full License 


\section{Abstract}

Background Apixaban is the most widely used direct oral anticoagulant in patients with chronic kidney disease (CKD). Data on the incidence of stroke and major bleeding after switching from warfarin to apixaban in patients with prevalent atrial fibrillation (AF) and CKD are limited.

Methods Warfarin users with stage 4-5 CKD not on dialysis and non-valvular AF prior to Jan 1,2012 were identified from the United States Data Renal System CKD dataset and individuals switching to apixaban from Jan 1,2012 -Dec 31, 2015 were identified. The incidence of stroke (ischemic or hemorrhagic), transient ischemic attack, or systemic thromboembolism and major bleeding events were estimated. Outcomes were compared between individuals switching to apixaban and those continuing warfarin using survival analyses with inverse probability treatment weighting. Individuals were censored at the time of anticoagulation discontinuation, loss of Medicare part D coverage, dialysis, kidney transplant, a $2^{\text {nd }}$ switch in anticoagulant class, or death.

Results 1762 individuals with advanced CKD and AF were initially on warfarin; 71 (4.0\%) switched to apixaban (57.8\% male, mean age 78.2 years ( $S D \pm 6.6)$, $78.9 \%$ white, mean $\mathrm{CHA}_{2} \mathrm{DS}_{2}$-VASc $5.0(\mathrm{SD} \pm 1.5)$, mean HAS-BLED 2.2 (SD \pm 0.5$)$ and 1691 (96.0\%) continued warfarin (47.6\% male, mean age 80.1 years (SD \pm 8.7 ), 87.9\% white, mean $\mathrm{CHA}_{2} \mathrm{DS}_{2}$-VASc 5.5 (SD \pm 1.6 ), mean HAS-BLED 2.5 (SD \pm 0.8$)$. The incidence of stroke in the apixaban switch and warfarin continuation groups were 0.02 / patient-year $(95 \% \mathrm{Cl} 0.002$ $0.12)$ and $0.06 /$ patient-year $(95 \% \mathrm{Cl} 0.05-0.07)(p=0.21)$. Incidence of major bleeding were 0.02 /patientyear $(95 \% \mathrm{Cl} 0.002-0.13)$ and $0.06(95 \% \mathrm{Cl} 0.03-0.04)(\mathrm{p}=0.44)$ in the switch and warfarin groups, respectively. In adjusted models, the risk of stroke (HR 0.27 (95\% $\mathrm{Cl} 0.04-1.99)$ ) and major bleeding (HR 0.41 (95\% $\mathrm{Cl} 0.06-3.02)$ ) trended lower in the apixaban switch compared to the warfarin continuation group.

Conclusions The incidence and risk of stroke and major bleeding trended lower in individuals with stage 4-5 CKD and prevalent AF who switched from warfarin to apixaban than individuals continuing warfarin. Our findings support a strategy of switching prevalent AF patients with late stage CKD from warfarin to apixaban. Additional studies including a larger number of events with a longer-duration of follow-up are needed to refine effect estimates.

\section{Introduction}

Atrial fibrillation (AF) and chronic kidney disease (CKD) commonly coexist ${ }^{1}$. Anticoagulation offers protection from thromboembolism but comes with a risk of major bleeding that is potentially lifethreatening. Compared to individuals with $\mathrm{AF}$ and without CKD, individuals affected by both conditions have up to a 3-fold increase in the risk of stroke related to $\mathrm{AF}^{2,3,4}$ and a 2-fold increase in the risk of major bleeding while receiving anticoagulation ${ }^{2}$. Data on how to best treat individuals with concomitant AF and CKD is needed to improve care. 
The vitamin K antagonist warfarin has been the cornerstone to anticoagulation since the 1950s especially in CKD as it is not cleared by the kidneys. In 2012 apixaban, a Factor Xa inhibitor with lower kidney clearance $(25 \%)$ relative to the other direct acting oral anticoagulants, was approved as the first direct factor Xa inhibitor ${ }^{5}$. Because apixaban demonstrated superior efficacy and safety compared with warfarin in the phase 3 clinical trials $s^{6,7,8}$, it has been rapidly adapted into clinical practice as a preferred anticoagulant $9,10,11$. Though the pivotal clinical trials of apixaban included patients with moderate stage 3 CKD they excluded patients with advanced CKD stage 4-5 and primarily enrolled patients with new onset (incident) AF rather than those with pre-existing (prevalent) AF ${ }^{6,7,8}$. Furthermore, to our knowledge, there are no randomized controlled data on the safety and efficacy of switching from warfarin to apixaban. In particular, the metabolism of apixaban by the kidneys, the comparative effectiveness of switching from warfarin to apixaban versus continuing on warfarin in preventing stroke and major bleeding outcomes in individuals with advanced CKD and prevalent $A F$ is an important and unanswered clinical question.

The aim of this study was therefore to evaluate the effectiveness and safety of switching from warfarin to apixaban in individuals with prevalent AF and advanced CKD stage 4-5 not on dialysis. Using real world data from the United States Data Renal System (USRDS) ${ }^{12}$ we compared risks of hemorrhagic and ischemic stroke and major bleeding in patients switching from warfarin to apixaban and patients continuing warfarin.

\section{Methods}

\section{Study Population}

Individuals with stage 4-5 CKD not on dialysis and prevalent non-valvular AF or atrial flutter on warfarin prior to Jan 1,2012 were identified from the USRDS ${ }^{12}$ CKD files. Prior to January 1, 2012, individuals without continuous Medicare Parts A\&B coverage for a year, a warfarin prescription within six months, or continuous Medicare Part D coverage for six months were excluded. Additional exclusion criteria included the following: valvular heart disease, dialysis start date prior to January 1,2012, or history of kidney transplantation (Fig. 1). The presence of any prescription claim for warfarin within the 6 months prior to Jan 1, 2012 was used to identify warfarin use since this criteria would include all stroke events, major bleeds, and deaths that occur before the next prescription refill and reduce the risk of bias towards a healthier population that would occur if clinical events resulted in discontinuation and the absence of subsequent prescriptions.

Individuals were followed from Jan 1,2012 through Dec 31, 2015 for warfarin to apixaban switching and endpoints. Switching to apixaban was defined by the presence of at least $1 \mathrm{claim}$ for apixaban during the study period January 1, 2012 - December 31, 2015. To be consistent with common prescribing patterns, anticoagulation discontinuation was defined for both the warfarin and apixaban switch groups using the 
date of the last available warfarin or apixaban prescription plus 90 days to account for a typical 3-month drug supply. Follow up was censored at the 2 nd switch in anticoagulant class.

Comorbidity diagnoses were based on the presence of at least one inpatient or two outpatient claims within 1 year prior to January 1, 2012 using condition-specific codes from the 9th revision of the International Statistical Classification of Diseases and Related Health Problems. Baseline characteristics and relevant medication prescriptions were retrieved from the institutional and physician claims of the non-ESRD database in the USRDS ${ }^{12}$. The $\mathrm{CHA}_{2} \mathrm{DS}_{2}$-Vasc (congestive heart failure, hypertension, age, diabetes, stroke, vascular history) ${ }^{13}$ was calculated along with the modified HAS-BLED score (hypertension, renal disease, liver disease, stroke, prior bleeding or predisposition to bleeding, age, medication, alcohol use $)^{14,15}$. The modified HAS-BLED score utilizes the original score but removes the component for labile INRs as INR values are not typically available in administrative data such as the USRDS. $^{15}$

The Partners Healthcare institutional review board approved the study (2016P001613/BWH) and ruled that informed consent was not necessary. This work was carried out in accordance with the declaration of Helsinki.

\section{Outcomes}

The primary effectiveness outcome was hospital admission for a composite of new stroke or embolism which was defined as a composite of ischemic stroke, transient ischemic attack, systemic thromboembolism, or hemorrhagic stroke ${ }^{16}$. The primary safety outcome was major bleeding defined as any bleeding resulting in death and occurring at a critical site (intracranial, intraocular, retroperitoneal, intra-articular, pericardial, airway), or resulting in hospitalization. ICD 9 and 10 codes were utilized (Supplemental Table 1). Patients were followed up to the date of death, dialysis, kidney transplantation, or on August 1, 2017, the last date for which mortality was available. In addition, follow-up was censored at the time of anticoagulation discontinuation, discontinuous Medicare part $\mathrm{D}$, dialysis, kidney transplant, or a 2nd switch in anticoagulant type.

\section{Statistical Analyses}

Poisson regression was used to estimate the incidence rates of stroke, the individual components of the stroke endpoint (hemorrhagic or ischemic), and major bleeding events. The primary analysis was conducted to determine the comparative effectiveness and safety of switching from warfarin to apixaban compared to continuing warfarin. Fine and Grey's models for competing risk survival analyses were used to compare the time to first all cause stroke or major bleeding event with death as competing risk between the two groups adjusted for potential covariates with an inverse probability treatment weighting (IPTW) approach ${ }^{17}$. Eight covariates were selected due to their potential to confound a clinician's decision to initiate treatment with apixaban and their association with mortality: age, sex, $\mathrm{CHA}_{2} \mathrm{DS}_{2}$-Vasc score, HAS-BLED score, history of stroke, history of bleeding, history of diabetes mellitus, history of heart disease (defined as either myocardial infarction, coronary artery disease, or congestive heart failure). 
Predicted probabilities for switching to apixaban (versus continuing warfarin) conditional on these 8 variables were used to calculate the standardized IPTW. For apixaban-switch patients, standardized weights were generated by taking the reciprocal of the estimated probability of switching to apixaban and multiplying it by unadjusted overall probability of apixaban treatment. For patients staying on warfarin, we took the reciprocal of the probability of not switching and multiplied it by the unadjusted probability of not switching. These weights were then used in the aforementioned primary analysis along with the robust sandwich variance estimator to account for potential dependency introduced by using IPTW. Results are reported in hazard ratios (HR) with 95\% confidence intervals (Cls). The follow up period started on the date of switch for warfarin and January 2, 2012 for the warfarin continuation group.

Due to the high mortality risk in advanced CKD, a sensitivity analysis was conducted to account for the potential effects of death from factors other than stroke or bleeding. Individuals who died within 90 days of January 1,2012 for the warfarin continue group and died within 90 days of the switch date for the apixaban switch group were excluded from the survival analyses. Fine and Grey's models were used to compare the time to first stroke or major bleeding event with death as competing risk (if death occurred after 90 days since the start date of the follow up period) between the two subgroups adjusted for potential covariates with IPTW described above. The SAS version 9.4 was used for all analyses. $\mathrm{P}<0.05$ was considered significant.

\section{Results}

\section{Baseline characteristics}

Out of 1762 individuals with advanced CKD with AF on warfarin, 71 (4.0\%) switched to apixaban 1691 (96.0\%) continued warfarin. The majority of warfarin to apixaban switching occurred in 2015 (Fig. 1). Compared with individuals continuing warfarin those switching to apixaban were more frequently male ( $57.8 \%$ vs. $47.6 \%)$, younger ( $78.2 \pm 6.6$ years vs. $80.1 \pm 8.7$ years), and less likely to be white $(78.9 \%$ vs. $87.9 \%$ ). Mean $\mathrm{CHA}_{2} \mathrm{DS}_{2}$-VASc score ( $5.0 \pm 1.5$ vs. $\left.5.5 \pm 1.6\right)$ and HAS-BLED scores (2.2 SD \pm 0.5 vs. $2.5 \pm$ 0.8 ) were lower in individuals switching to apixaban however the distribution between CKD stage 4 and 5 was similar (Table 1). 
Table 1

Baseline Characteristics of the Study Population

\section{Characteristic}

Warfarin Continue

1691 (96.0\%)

\section{Apixaban Switch}

$71(4.0 \%)$

\section{Demographics}

\begin{tabular}{lll} 
Age, years & $80.1 \pm 8.7$ & $78.2 \pm 6.6$ \\
Sex, male & $804(47.6)$ & $41(57.8)$ \\
Race & $1486(87.9)$ & $56(78.9)$ \\
White & $134(7.9)$ & $<11$ \\
Black & $25(1.5)$ & $<11$ \\
\hline Asian & $46(2.8)$ & $<11$ \\
\hline Other* & & $68(95.8)$ \\
\hline Clinical Factors & $1582(93.55)$ & $<11$ \\
\hline CKD stage 4 & $109(6.45)$ & $43(60.6)$ \\
\hline CKD stage 5 & $962(56.9)$ & $68(95.8)$ \\
\hline Diabetes & $1608(95.1)$ & $35(49.3)$ \\
\hline Hypertension & $1098(64.9)$ & $55(77.5)$ \\
\hline CHF & $1278(75.6)$ & $11(15.5)$ \\
\hline HLD & $428(25.3)$ & $46(64.8)$ \\
\hline CVA & $1097(64.9)$ & $<11$ \\
\hline CAD & $82(4.9)$ & $<11$ \\
\hline Bleeding History & $68(4.0)$ & \\
\hline Heavy ETOH Use & &
\end{tabular}

Continuous variables reported as Mean $\pm \mathrm{SD}$, categorical variables presented as $\mathrm{N}(\%)$

*Other race $=$ Native American or Alaskan, other, and unknown.

**Modified utilizing the original score except labile INRs

$\mathrm{CHF}=$ congestive heart failure; $\mathrm{CAD}=$ coronary artery disease; $\mathrm{CVA}=$ cerebrovascular attack which is a composite of ischemic stroke and transient ischemic attack; Heavy ETOH = Alcohol use (defined at > 15 drinks/week); ICH = intracranial hemorrhage. $\mathrm{CHA}_{2} \mathrm{DS}_{2}$-Vasc = congestive heart failure, hypertension, age, diabetes, stroke, vascular history); HAS-BLED (modified) = hypertension, renal disease, liver disease, stroke, prior bleeding or predisposition to bleeding, age, medication, alcohol use. 


\begin{tabular}{|lll|}
\hline Characteristic & Warfarin Continue & Apixaban Switch \\
& $\mathbf{1 6 9 1}(\mathbf{9 6 . 0 \% )}$ & $\mathbf{7 1}(\mathbf{4 . 0 \% )}$ \\
\hline ICH & $18(1.1)$ & $<11$ \\
\hline CHA2DS2-VASC & $5.5 \pm 1.6$ & $5.0 \pm 1.5$ \\
\hline HASBLED** & $2.5 \pm 0.8$ & $2.2 \pm 0.5$ \\
\hline
\end{tabular}

Continuous variables reported as Mean \pm SD, categorical variables presented as $\mathrm{N}(\%)$

*Other race $=$ Native American or Alaskan, other, and unknown .

**Modified utilizing the original score except labile INRs

$\mathrm{CHF}=$ congestive heart failure; $\mathrm{CAD}=$ coronary artery disease; $\mathrm{CVA}=$ cerebrovascular attack which is a composite of ischemic stroke and transient ischemic attack; Heavy ETOH = Alcohol use (defined at > 15 drinks/week); ICH = intracranial hemorrhage. $\mathrm{CHA}_{2} \mathrm{DS}_{2}$-Vasc = congestive heart failure, hypertension, age, diabetes, stroke, vascular history); HAS-BLED (modified) = hypertension, renal disease, liver disease, stroke, prior bleeding or predisposition to bleeding, age, medication, alcohol use.

\section{Incidence of all cause stroke and major bleeding in apixaban switch and warfarin continuation groups}

In unadjusted analyses, the incidence rate of stroke was numerically but not significantly lower in the apixaban switch $(0.02$ / patient-year, $95 \% \mathrm{Cl} 0.002-0.12)$ compared with the warfarin continuation groups $(0.06 /$ patient-year, $95 \% \mathrm{Cl} 0.05-0.07, \mathrm{p}=0.21)$. Results were consistent when broken down into the individual components ischemic stroke, systemic thromboembolism, hemorrhagic stroke, and TIA. Of the 303 events in the warfarin continuation group there were 250 ischemic stroke events, 29 systemic thromboembolism events, 22 hemorrhagic stroke events, and 2 TIA events. In the apixaban switch group only one event, an ischemic stroke, occurred (Supplemental Table 2). There were 1294 participants censored (337 died, 92 started dialysis, 10 switched back to warfarin from apixaban, and the remainder reached the end of follow up).

The incidence of major bleeding in the two groups was $0.018 /$ patient year $(95 \% \mathrm{Cl} 0.002-0.13)$ and 0.05 $(95 \% \mathrm{Cl} 0.03-0.04, \mathrm{p}=0.44)$, respectively (Table 2). There were 202 events of major bleeding: 201 and 1 occurred in the warfarin continuation and apixaban switch groups respectively. There were 1225 participants censored (290 died, 92 started dialysis, 10 switched back to warfarin from apixaban, and the remainder reached the end of follow up). 
Table 2

Incidence of All Cause Stroke and Major Bleeding in Patients with Prevalent Atrial Fibrillation on Warfarin who Switch to Apixaban versus Patients who Continue on Warfarin

Warfarin Continue

Apixaban Switch

$P$ value

Stroke

Incidence, patient year $(95 \% \mathrm{Cl})$

$0.061(0.054-0.068)$

$0.020(0.002-0.123)$

0.211

Major Bleeding

Incidence, patient year $(95 \% \mathrm{Cl})$

$0.045(0.033-0.044)$

$0.018(0.002-0.125)$

0.438

Individuals were censored at anticoagulation discontinuation, discontinuous Medicare part D, dialysis, kidney transplant, a 2nd switch in anticoagulant, or death. Poisson regression was used to estimate the incidence rates of stroke (hemorrhagic or ischemic) and major bleeding events.

\section{Risk of stroke and major bleeding in apixaban switch and warfarin continuation groups}

Covariates were well balanced between groups using ITPW noting the standardized differences for HASBLED and age were -0.23 and -0.18 respectfully (Supplemental Table 3 ). In competing-risks survival models (with death as the competing risk) using the IPTW approach, point estimates were consistent with numerically lower risks of both all-cause stroke ( $\mathrm{HR} 0.27,95 \% \mathrm{Cl} 0.04-1.99, \mathrm{p}=0.20)$ and major bleeding (HR $0.41,95 \% \mathrm{Cl} 0.06-3.02, \mathrm{p}=0.38$ ) in the apixaban switch compared to the warfarin continuation group (Table 3) but did not achieve significance.

Table 3

Adjusted Risk of All Cause Stroke and Major Bleeding in Patients with Atrial Fibrillation on Warfarin who Switch to Apixaban versus Continue on Warfarin

Risk Estimate $(95 \% \mathrm{Cl}) \quad$ P value

All Cause Stroke

Adjusted HR $(95 \% \mathrm{Cl})$

$0.27(0.04-1.99)$

0.20

Major Bleeding

Adjusted HR $(95 \% \mathrm{Cl})$

$0.41(0.06-3.02)$

0.38

Inverse probability treatment weighting (IPTW) adjusted survival analyses with death as competing risk were used to compare the time to first all cause stroke or major bleeding event between groups. The eight covariates used in the IPTW model were: age, sex, $\mathrm{CHA}_{2} \mathrm{DS}_{2}$-Vasc score, HAS-BLED score, history of stroke, history of bleeding, history of diabetes mellitus, history of heart disease (defined as either myocardial infarction, coronary artery disease, or congestive heart failure). Individuals were censored at anticoagulation discontinuation, discontinuous Medicare part D, dialysis, kidney transplant, a 2nd switch in anticoagulant, or death.

$\mathrm{HR}=$ hazard ratio; $\mathrm{Cl}=$ confidence interval 


\section{Risk of stroke and major bleeding in apixaban switch and warfarin continue groups: sensitivity analyses}

Sensitivity analyses removing individuals who died within 90 days of January 1,2012 for the warfarin continue group and within 90 days of the switch date for the apixaban switch group results were qualitatively similar to the primary analysis. Non-significant trends consistent with reduced risks of stroke and bleeding in the apixaban switch compared to warfarin continuation groups were observed (Supplemental Table 4).

\section{Discussion}

Several studies suggest that apixaban is a safer or more effective alternative to warfarin for patients with CKD and AF. However, whether patients who are stable on warfarin ought to be switched to apixaban is uncertain. Our study directly addresses the comparative safety and efficacy in switching individuals with advanced CKD not on dialysis and prevalent AF from warfarin to apixaban versus a strategy of continuing warfarin. Using real world evidence from the USRDS database events of stroke and bleeding were compared between individuals switching to apixaban and continuing warfarin. Although patients in this study, had used warfarin for varying periods of time, results show a trend toward improved outcomes in reducing the risk of all-cause stroke and major bleeding in the group switching to apixaban compared with continuing on warfarin. These findings are hypothesis-generating and supported by the benefits observed in subset of advanced CKD and prevalent AF patients from the phase 3 clinical trials of apixaban $^{6,7,8}$. In the context of apixaban's introduction to the US market in 2012, this study spans the first three years of its use in clinical practice adding early evidence to the potential benefits of switching anticoagulation agents as suggested by other cohort studies and reviews ${ }^{9,10}$.

The strengths of the study lie within the identified patient population and methodology. By utilizing a national data collection system, a sufficient sample of individuals with stage 4-5 CKD and prevalent AF on warfarin was identified including 71 individuals switched to apixaban. However, the overall proportion of individuals switching to apixaban was low ( $4 \%$ of the total cohort). Several factors contributed to this low proportion. Most importantly, we required warfarin prescription for at least six months prior to 2012. Therefore, patients who were started on warfarin after January 1st, 2012 (and potentially switched to apixaban later) could not be captured. In addition, patients who developed CKD and/or AF between 2012 and 2015 are not included in this analysis. Moreover, apixaban was recently approved by the FDA in 2012 and changes in prescribing patterns often lag behind approval as physicians become accustomed to a new option. In fact, the majority of individuals were switched from warfarin to apixaban after 2014. However, the overall cohort size declines over the course of the study timeframe due to censoring from for death, dialysis, or kidney transplant, second anticoagulation switch, or anticoagulation discontinue. Additionally, evaluation of individuals with kidney impairment from the clinical trials were not published until $2016^{7,8}$ and a reversal agent was not approved until 2018. Since these two events occurred after 
completion of the study follow up period, the low proportion of apixaban switch patients in such a highrisk patient population is not unexpected.

The short duration on apixaban and small number of events in the apixaban switch group are notable limitations. Since individuals were evaluated at any time for switching, the follow up period for evaluating outcomes varied with the longest duration possible of approximately 3 years. Though there were 304 and 202 events of stroke and major bleeding combined in both groups only 1 event in each outcome occurred in the apixaban switch group. Additional studies of larger cohorts with longer follow up are needed to validate these findings. Power to detect significant effects was limited due to the low number of apixaban switch patients. However, these data are reassuring and suggest better outcomes with switching despite stable use of warfarin.

In summary, our data provides initial evidence to healthcare providers to inform anticoagulation choice with high risk patients with AF and advanced CKD. Although further study is needed, these findings suggest that the risks and benefits of different anticoagulation options may favor a switch to apixaban for CKD patients already using warfarin.

\section{List Of Abbreviations}

chronic kidney disease (CKD)

atrial fibrillation (AF)

inverse probability treatment weighting (IPTW)

United States Data Renal System (USRDS)

hazard ratios $(H R)$

confidence intervals (Cls)

\section{Declarations}

Ethics approval and consent to participate: The Partners Healthcare institutional review board approved the study (2016P001613/BWH) and ruled that informed consent was not necessary.

Consent for publication: All authors give consent for publication. The corresponding author has read the BMC journal policies on author responsibilities and submits this manuscript in accordance with those policies. The results/data/figures in this manuscript been not been published and are not under consideration for publication elsewhere.

Availability of data and materials: The data reported here have been supplied by the United States Renal Data System (USRDS). The interpretation and reporting of these data are the responsibility of the 
author(s) and in no way should be seen as an official policy or interpretation of the U.S. government.

Competing interests: Dr. Charytan reports personnel fees from Janssen, Astra Zeneca, Gilead, Amgen, NovoNordisk, Merck and Glaxo Smith Kline and research funding from Janssen, Amgen, Gilead and NovoNordisk. Dr. Garlo is employed by Alexion. The remaining authors have not conflicts to declare.

Funding: American Heart Association AWRP Mentored Clinical \& Population Research Award 17MCPRP33661217 titled "Safety and Efficacy of Non Vitamin K Novel Antagonists Compared with Warfarin in Patients with Atrial Fibrillation and Chronic Kidney Disease."

Authors Contributions: KG contributed the study conception, design of work, interpretation of data, and manuscript composition; TM contributed data acquisition, design of work, interpretation of data, and manuscript revision; LB contributed database management and programming; WW contributed biostatistical expertise and modeling; DC contributed design of work, interpretation of data, and manuscript drafting.

Acknowledgments: We thank Dr. Julian Seifter, Dr. David Bates, and Dr. Christopher Cannon for their clinical research expertise and patient centric approach to anticoagulation management.

\section{References}

1. Lau YC, Proietti M, Guiducci E, Blann AD, Lip GYH: Atrial fibrillation and thromboembolism in patients with chronic kidney disease. J Am Coll Cardiol 68: 1452-1464, 2016

2. Olesen JB, Lip GYH, Kamper AL, Hommel K, Køber L, Lane DA, Lindhardsen J, Gislason GH, TorpPedersen C: Stroke and bleeding in atrial fibrillation with chronic kidney disease. N Engl J Med 367: 625-635, 2012

3. Dad T, Weiner DE. Stroke and Chronic Kidney Disease: Epidemiology, Pathogenesis, and Management Across Kidney Disease Stages. Semin Nephrol. 2015;35(4):311-322.

4. Ninomiya T, Perkovic V, Verdon C, Barzi F, Cass A, Gallagher M, et al. Proteinuria and stroke: a metaanalysis of cohort studies. Am J Kidney Dis. 2009;53:417-25.

5. Apixaban (Eliquis) [package insert], Princeton, NJ, Bristol-Myers Squibb Company, and New York, Pfizer Inc., 2012

6. Granger CB, Alexander JH, McMurray JJ, Lopes RD, Hylek EM, Hanna M, Al-Khalidi HR, Ansell J, Atar D, Avezum A, Bahit MC, Diaz R, Easton JD, Ezekowitz JA, Flaker G, Garcia D, Geraldes M, Gersh BJ, Golitsyn S, Goto S, Hermosillo AG, Hohnloser SH, Horowitz J, Mohan P, Jansky P, Lewis BS, LopezSendon JL, Pais P, Parkhomenko A, Verheugt FW, Zhu J, Wallentin L; ARISTOTLE Committees and Investigators: Apixaban versus warfarin in patients with atrial fibrillation. N Engl J Med 365: 981992, 2011

7. Alexander JH, Andersson U, Lopes RD, Hijazi Z, Hohnloser SH, Ezekowitz JA, Halvorsen S, Hanna M, Commerford P, Ruzyllo W, Huber K, Al-Khatib SM, Granger CB, Wallentin L; Apixaban for Reduction of 
Stroke and Other Thromboembolic Complications in Atrial Fibrillation (ARISTOTLE) Investigators: Apixaban $5 \mathrm{mg}$ twice daily and clinical outcomes in patients with atrial fibrillation and advanced age, low body weight, or high creatinine: A secondary analysis of a randomized clinical trial. JAMA Cardiol 1: 673-681, 2016

8. Hijazi Z, Hohnloser SH, Andersson U, Alexander JH, Hanna M, Keltai M, Parkhomenko A, Lo'pezSendo'n JL, Lopes RD, Siegbahn A, Granger CB, Wallentin L: Efficacy and safety of apixaban compared with warfarin in patients with atrial fibrillation in relation to renal function over time: Insights from the ARISTOTLE randomized clinical trial. JAMA Cardiol 1: 451-460, 201632.

9. Pelliccia F, Rosanio S,MarazziG, Poggi S, TanzilliA,GrecoC,Gaudio C, Rosano G: Efficacy and safety of novel anticoagulants versus vitamin $\mathrm{K}$ antagonistsin patients with mild and moderateto severe renal insufficiency: Focus on apixaban. Int J Cardiol 225: 77-81, 2016 33. KimachiM, Furukawa TA, Kimachi K, Goto Y, Fukuma S, Fukuhara S: Direct oral anticoagulants versus warfarin for preventing stroke and systemic embolic events among atrial fibrillation patients with chronic kidney disease. [Review] Cochrane Database Syst Rev 11: CD011373, 2017

10. Shin JI, Secora A, Alexander GC, Inker LA, Coresh J, Chang AR, Grams ME: Risks and benefits of direct oral anticoagulants across the spectrum of GFR among incident and prevalent patients with atrial fibrillation. Clin J Am Soc Nephrol 13: 1144-1152, 2018

11. January CT, Wann LS, Alpert JS, Calkins H, Cigarroa JE, Cleveland JC Jr., Conti JB, Ellinor PT, Ezekowitz MD, Field ME, Murray KT, Sacco RL, Stevenson WG, Tchou PJ, Tracy CM, Yancy CW; American College of Cardiology/American Heart Association Task Force on Practice Guidelines: 2014 AHA/ACC/HRS guidelineforthe management of patientswith atrial fibrillation: A report of the American College of Cardiology/American Heart Association Task Force on Practice Guidelines and the Heart Rhythm Society. J Am Coll Cardiol 64: e1-e76, 2014

12. U.S. Renal Data System. 2019 USRDS annual data report: Epidemiology of kidney disease in the United States. National Institutes of Health, National Institute of Diabetes and Digestive and Kidney Diseases, Bethesda, MD, 2019.

13. Thigpen JL, Dillon C, Forster KB, Henault L, Quinn EK, Tripodis Y, Berger PB, Hylek EM, Limdi NA: Validity of international classification of disease codes to identify ischemic stroke and intracranial hemorrhage among individuals with associated diagnosis of atrial fibrillation. Circ Cardiovasc Qual Outcomes 8: 8-14, 2015

14. Tsu LV, Berry A, Wald E, Ehrlich C: Modified HAS-BLED score and risk of major bleeding in patients receiving dabigatran and rivaroxaban: A retrospective, case-control study. Consult Pharm 30: 395402, 2015

15. Donze' J,RodondiN,WaeberG,MonneyP,CornuzJ,AujeskyD: Scores to predict major bleeding risk during oral anticoagulation therapy: A prospective validation study. Am J Med 125: 1095-1102, 201246.

16. Shaw PA: Use of composite outcomes to assess risk-benefit in clinical trials. Clin Trials 15: 352-358, 2018 
17. Xu S, Ross C, Raebel MA, Shetterly S, Blanchette C, Smith D. Use of stabilized inverse propensity scores as weights to directly estimate relative risk and its confidence intervals. Value in Health. 2010;13(2):273-277.

\section{Figures}

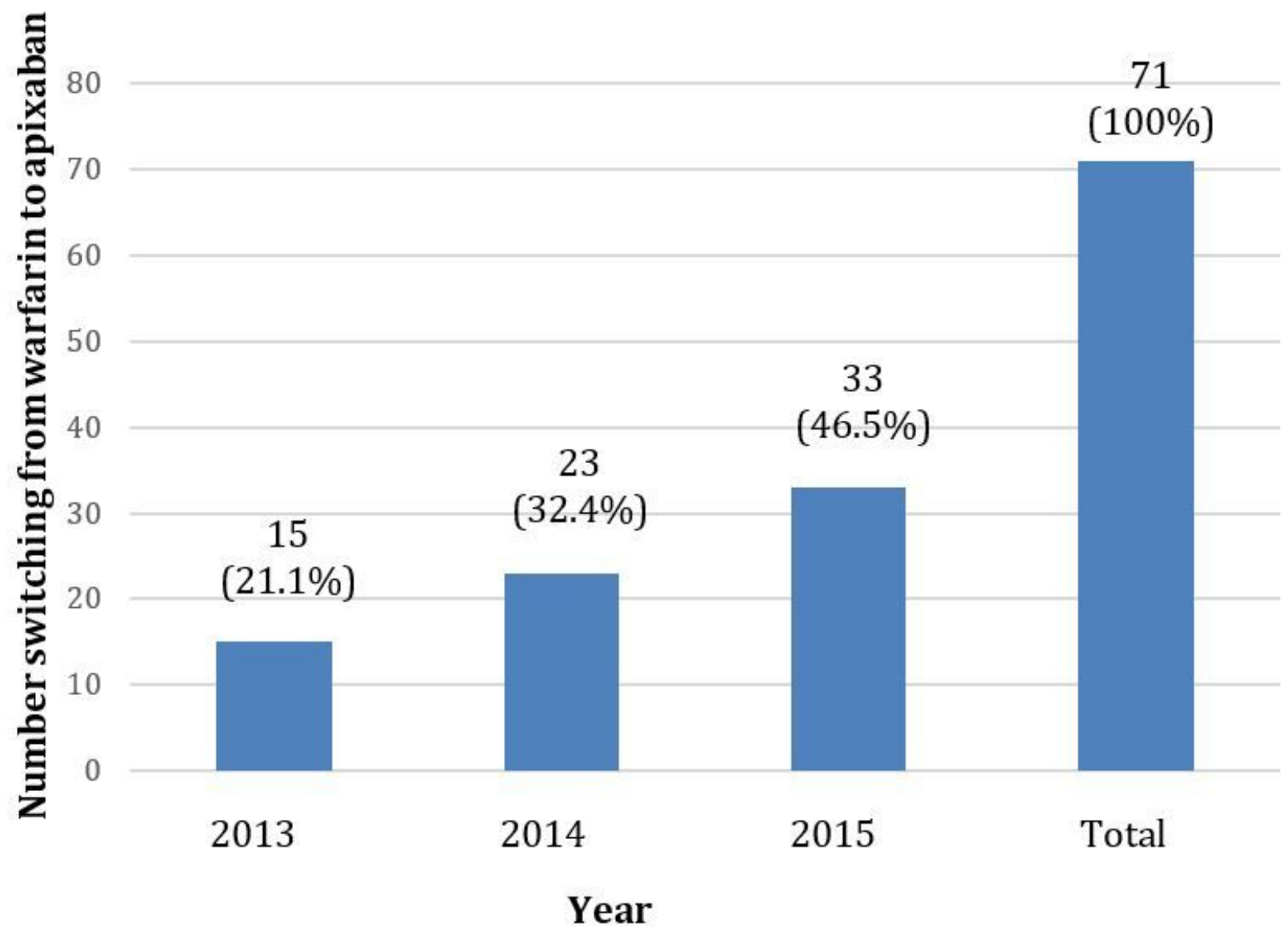

\section{Figure 1}

Warfarin to apixaban switch in CKD stage 3-4 with prevalent atrial fibrillation from 2012-2015 in the United States Data Renal System among patients who were on warfarin for at least six months prior to January 1 st, 2012

\section{Supplementary Files}

This is a list of supplementary files associated with this preprint. Click to download.

- CKDApixSupplemental.docx 